\title{
UNKNOTTING IN CODIMENSION ONE
}

\author{
L. S. HUSCH ${ }^{1}$
}

Two PL (=Piecewise linear) embeddings $f, g$ of a polyhedron $X_{1}$ into a polyhedron $X_{2}$ are equivalent if there exists a PL-homeomorphism $h$ of $X_{2}$ onto $X_{2}$ such that $h f=g$. Let $Y_{i}$ be a subpolyhedron of $X_{i}, i=1,2$; a PL-map $f$ of the pair $\left(X_{1}, Y_{1}\right)$ into $\left(X_{2}, Y_{2}\right)$ is a PL-map $f: X_{1} \rightarrow X_{2}$ such that $f^{-1}\left(Y_{2}\right)=Y_{1}$. Two PL-embeddings, $f, g$, of the pair $\left(X_{1}, Y_{1}\right)$ into $\left(X_{2}, Y_{2}\right)$ are equivalent if there exists a PL-homeomorphism $h$ of the pair $\left(X_{2}, Y_{2}\right)$ onto $\left(X_{2}, Y_{2}\right)$ such that $h f=g$. Two PL-embeddings, $f, g$ of the pair $\left(X_{1}, Y_{1}\right)$ into $\left(X_{2}, Y_{2}\right)$ such that $f\left|Y_{1}=g\right| Y_{1}$ are equivalent relative to $Y_{2}$ if there exists a PL-homeomorphism $h$ of the pair $\left(X_{2}, Y_{2}\right)$ onto itself such that $h f=g$ and $h \mid Y_{2}$ is the identity map.

Let $S^{p}, B^{p}$ denote the PL-p-sphere and PL-p-cell respectively. Zeeman [11] has shown that any two PL-embeddings of $S^{p}$ into $S^{n}$ and any two PL embeddings of $\left(B^{p}, \partial B^{p}\right.$ (=) boundary of $\left.B^{p}\right)$ ) into $\left(B^{n}, \partial B^{n}\right)$ are equivalent provided $n-p \geqq 3$. Let $X^{p-1}$ be a compact polyhedron and $v * X^{p-1}$ be the cone over $X^{p-1}$. Lickorish [7] generalized Zeeman's work to show that any two PL-embeddings of $\left(v * X^{p-1}, X^{p-1}\right)$ into $\left(B^{n}, \partial B^{n}\right)$ are equivalent relative to $\partial B^{n}$ provided $n-p \geqq 3$.

Let $M^{n+1}, N^{n}$ be $(n+1)$ - and $n$-dimensional closed PL-manifolds. In this paper, we investigate embeddings of $\left(v * N^{n}, N^{n}\right)$ into $\left(w * M^{n+1}, M^{n+1}\right)$ which take $v$ to $w$. If the embedding $f$ of $N^{n}$ into $M^{n+1}$ induces isomorphisms of fundamental groups, we show that $w * M^{n+1}$ collapses to $f\left(v * N^{n}\right)$ for $n \geqq 5$. If, in addition, $f$ and $g$ are two locally unknotted embeddings which agree on $N^{n}$, then we can apply results of Akin [1] to obtain that $f$ and $g$ are equivalent relative to $M$. As an application of this result we obtain a covering concordance theorem.

We assume familiarity with either [5] or [12]. We express our gratitude to Henry Edwards, John Hollingsworth and Tom Kyrouz for their help and comments.

1. Collapsing to embedded cones. Suppose $f:\left(B^{p}, \partial B^{p}\right) \rightarrow\left(B^{n}, \partial B^{n}\right)$ is a PL-embedding of the pair. One of the essential steps in Zeeman's proof [11] was to show that $B^{n}$ collapses to $f\left(B^{p}\right)$ if $n-p \geqq 3$. In [6], it was shown that $B^{n}$ collapsed to $f\left(B^{p}\right)$ if $n=p+1 \geqq 6$. As noted in

Received by the editors December 16, 1968.

${ }^{1}$ Research supported in part by National Science Foundation grant GP-8615. 
[6], collapsing in either $n=4$ or 5 implies the Schönflies Conjecture. We generalize as follows.

TheOREM 1. Let $M^{n+1}, N^{n}$ be $(n+1)$ - and $n$-dimensional closed connected PL-manifolds, $n \geqq 5$. Let $f$ be a PL-embedding of the pair $\left(v * N^{n}, N^{n}\right)$ to $\left(w * M^{n+1}, M^{n+1}\right)$ such that $f(v)=w, f\left(N^{n}\right)$ separates $M^{n+1}$, and $f$ induces an isomorphism $f_{*}: \Pi_{1}\left(N^{n}\right) \rightarrow \Pi_{1}\left(M^{n+1}\right)$; then $w * M^{n+1}$ collapses to $f\left(v * N^{n}\right)$.

Let $L, K$ be triangulations of $v * N^{n}, w * M^{n+1}$ respectively such that $f: L \rightarrow K$ is simplicial. Let $Q=\operatorname{st}(a, K)$, which we may suppose does not intersect $M^{n+1}$, and let $R=N\left(f\left(v * N^{n}\right) \cap \mathrm{cl}\left(w * M^{n+1}-Q\right)\right.$, $\left.\left(K-\mathrm{st}^{\circ}(a, K)\right)^{\prime \prime}\right)$, - i.e. $R$ is the second derived neighborhood of $f\left(v * N^{n}\right) \cap \mathrm{cl}\left(w * M^{n+1}-Q\right)$ in the complement of the open star of $a$ in $K$.

Lemma 1. $R \cup Q$ collapses to $Q$.

Proof. Note that $R$ is a regular neighborhood of $f\left(v * N^{n}\right)$ $\cap \mathrm{cl}\left(w * M^{n+1}-Q\right)$ in the PL-manifold $\operatorname{cl}\left(w * M^{n+1}-Q\right)$. Hence $R \cup Q$ collapses to $f\left(v * N^{n}\right) \cup Q$ which clearly collapses to $Q$.

The following lemma follows from Cohen [3].

Lemma 2. The triple $\left(R, R \cap M^{n+1}, R \cap Q\right)$ is PL-homeomorphic to the triple $\left(N^{n} \times I \times I, N^{n} \times I \times 0, N^{n} \times I \times 1\right)$ where $I=[0,1]$.

Lemma 3. Let $S_{\boldsymbol{i}}$ be a component of $S=\mathrm{Cl}\left(w * M^{n+1}-(R \cup Q)\right)$. The following homomorphisms induced by inclusion are isomorphisms.

(1) $\Pi_{1}\left(S_{1} \cap M^{n+1}\right) \rightarrow \Pi_{1}\left(M^{n+1}\right)$

(2) $\Pi_{1}\left(f\left(v * N^{n}\right) \cap R\right) \rightarrow \Pi_{1}(R \cup S)$

(3) $\Pi_{1}\left(S_{i}\right) \rightarrow \Pi_{1}(R \cup S)$

(4) $\Pi_{1}\left(S_{i} \cap M^{n+1}\right) \rightarrow \Pi_{1}\left(S_{i}\right)$

(5) $\Pi_{1}\left(S_{i} \cap Q\right) \rightarrow \Pi_{1}\left(S_{i}\right)$

Proof. (1) Since $\Pi_{1}\left(f N^{n}\right) \rightarrow \Pi_{1}\left(M^{n+1}\right)$ is an isomorphism, $\Pi_{1}\left(R \cap M^{n+1}\right) \rightarrow \Pi_{1}\left(M^{n+1}\right)$ is an isomorphism. From Lemma 2, $\left(R \cup S_{i}\right) \cap M^{n+1}$ is PL-homeomorphic to $S_{i} \cap M^{n+1}$; hence $\Pi_{1}\left(S_{i} \cap M^{n+1}\right)$ $\rightarrow \Pi_{1}\left(M^{n+1}\right)$ is onto. Let $g: S^{1} \rightarrow S_{i} \cap M^{n+1}$ represent some element of $\Pi_{1}\left(S_{1} \cap M^{n+1}\right)$ such that $g$ can be extended to $g: D^{2} \rightarrow M^{n+1}$. We may asssme that $g$ is a PL-embedding which is in general position with respect to $f N^{n}$. Hence $f N^{n} \cap g D^{2}$ is a finite collection of disjoint simple closed curves. Since $\Pi_{1}\left(f N^{n}\right) \rightarrow \Pi_{1}\left(M^{n+1}\right)$ is an isomorphism, by the classical technique of "exchanging and popping" disks we can assume $f N^{n} \cap g D^{2}=\varnothing$ and hence $g D^{2} \subseteq S_{i}$. 
(2) follows from the following commutative diagram:

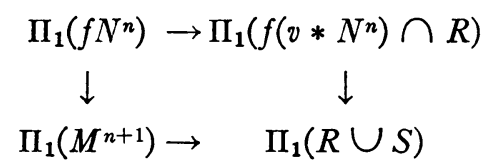

(3) follows from an argument similar to (1) and (4) follows from the following commutative diagram:

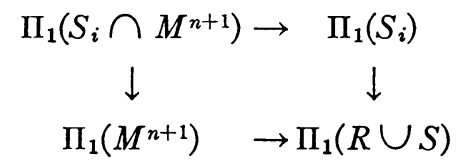

The arguments for (5) follow a similar pattern.

Lemma 4. $S$ is an h-cobordism between $S \cap Q$ and $S \cap M^{n+1}$.

Proof. Let $\tilde{X}$ denote the universal cover of $X$ and let $p: \tilde{X} \rightarrow X$ be the natural map. If $Y \subset X$ and $\Pi_{1} Y \rightarrow \Pi_{1} X$ is an isomorphism, then $p^{-1} Y=\tilde{Y}$.

Consider the triple $\left(\tilde{R} \cup \tilde{S}, \tilde{R} \cup(S \cap Q)^{\sim},((R \cup S) \cap Q)^{\sim}\right)$. Clearly the relative homology groups $H_{k}(\tilde{R} \cup \tilde{S},((R \cup S) \cap Q) \sim)$ and $H_{k}\left(\tilde{R} \cup(S \cap Q)^{\sim},((R \cup S) \cap Q) \sim\right)$ are zero for all $k$. By exactness of the homology sequence of the triple, $H_{k}\left(\tilde{R} \cup \widetilde{S}, \tilde{R} \cup(S \cap Q)^{\sim}\right)=0$ for all $k$. By excision, $H_{k}\left(\tilde{S},(\tilde{R} \cap \tilde{S}) \cup(S \cap Q)^{\sim}\right)=0$ for all $k$.

Consider the triple $(\tilde{S},(\tilde{R} \cap \tilde{S}) \cup(S \cap Q) \sim,(S \cap Q) \sim)$. From Lemma 2 , it follows that $H_{k}\left(\widetilde{S},(S \cap Q)^{\sim}\right)=0$ for all $k$. Hence $\Pi_{k}(S, S \cap Q)$ $=\Pi_{k}(\tilde{S},(S \cap Q) \sim)=0$ for all $k$ by the Hurewicz Theorem and Lemma 3. Therefore $S \cap Q$ is a deformation retract of $S$. Similarly, $S \cap M$ is a deformation retract of $S$.

Proof of Theorem 1. From [9], the Whitehead torsion $\tau\left(w * M^{n+1}, Q\right)=\tau\left(w * M^{n+1}, R \cup Q\right)+i_{*} \tau(R \cup Q, Q)=0$ since $w * M^{n+1}$ collapses to $Q$. From Lemma $1, \tau(R \cup Q, Q)=0$ and hence $i_{*} \tau(R \cup Q, Q)=0$. Therefore $\tau(S, Q \cap S)=\tau(S,(R \cup Q) \cap S)$ $=\tau\left(w * M^{n+1}, R \cup Q\right)=0$. By the $s$-cobordism Theorem [10], $S$ is PL-homeomorphic to $(S \cap Q) \times I$. Hence $S$ collapses to $S \cap Q$ and the theorem follows.

Example. If, in Theorem 1 , the condition that $f_{*}: \Pi_{1}\left(N^{n}\right)$ $\rightarrow \Pi_{1}\left(M^{n+1}\right)$ is not an isomorphism is not assumed, the conclusion may not be necessarily true. Consider the following example. Let $\left(B^{n+2}, B^{n}\right)$ be a locally unknotted, knotted ball pair such that $\left(\partial B^{n+2}, \partial B^{n}\right)$ is unknotted. Such ball pairs exist for $n \geqq 1$. Let $L$ be a regular neighborhood of $B^{n}$ in $B^{n+2}$ such that $L \cap \partial B^{n+2}$ is a regular neighborhood of $\partial B^{n}$ in $\partial B^{n+2}$. Let $N=\partial\left(L \cap \partial B^{n+2}\right)$, which is PL- 
homeomorphic to $S^{n-1} \times S^{1}$. Since $L$ is a PL- $(n+2)$-cell, there is a PL-homeomorphism $h: L \rightarrow v * \partial L$. Consider $h^{-1}:(v * h N, h N)$ $\rightarrow\left(B^{n+2}, \partial B^{n+2}\right)$. Note that $h^{-1}(v * h N)$ separates $B^{n+2}$ into two components with one closure $h^{-1}\left(v * h\left(L \cap \partial B^{n+2}\right)\right)$. If $B^{n+2}$ collapses to $h^{-1}(v * h N)$, then $B^{n+2}$ collapses to $h^{-1}\left(v * h\left(L \cap \partial B^{n+2}\right)\right)$ which in turn collapses to $B^{n}$. Since $\left(B^{n+2}, B^{n}\right)$ is locally unknotted, it follows from a proof similar to Zeeman's unknotting theorem [11] that $\left(B^{n+2}, B^{n}\right)$ is unknotted. Hence $B^{n+2}$ does not collapse to $h^{-1}(v * h N)$.

Let $f, M^{n+1}, N^{n}$ be as in Theorem 1. $f$ is said to be locally unknotted if for each point $p \in w * f N^{n}, p \neq w$, there exists a closed neighborhood $U$ of $p$ in $w * M^{n+1}$ such that $\left(U, U \cap f N^{n}\right)$ is an unknotted PL-ball pair.

The following theorem now follows from Akin [1] and Theorem 1.

Theorem 2. Let $f, M^{n+1}, N^{n}$ be as in Theorem 1 and suppose that $f$ is locally unknotted; then there exists a PL-homeomorphism $h: w * M^{n+1}$ $\rightarrow w * M^{n+1}$ such that $h \mid M^{n+1}$ is the identity and $h f=\hat{f}$ where $\hat{f}$ is the standard conical extension of $f \mid N^{n}$.

2. Covering concordances. Let $M$ and $N$ be compact PL-manifolds and let $f: N \rightarrow M$ be a PL-embedding; $f$ is proper if $f^{-1}(\partial M)=\partial N$. A PL-concordance of $N$ in $M$ is a proper PL-embedding $F: N \times I$ $\rightarrow M \times I$; an ambient PL-concordance of $M$ is a proper PL-homeomorphism $H: M \times I \rightarrow M \times I$ which is the identity on $M \times 0$. $H$ covers $F$ if $H\left(F_{0} \times 1\right)=F$ where $F_{0}=F \mid N \times 0$.

Hudson [4] showed that any PL-concordance of $N$ in $M$ can be covered by an ambient concordance of $M$ if codimension $N \leqq 3$. Lickorish and Siebenmann [8] extended these results in codimension $\leqq 3$ to the case when $N$ is a polyhedron.

Theorem 3. Let $M^{n+1}, N^{n}$ be $(n+1)$ - and $n$-dimensional closed connected PL-manifolds, $n \geqq 5$. Let $F$ be a locally unknotted concordance of $N^{n}$ in $M^{n+1}$ such that $F$ induces an isomorphism $F_{*}: \Pi_{1}\left(N^{n} \times I\right)$ $\rightarrow \Pi_{1}\left(M^{n+1} \times I\right)$; then $F$ can be covered by an ambient concordance of $M$.

Proof. Let

$$
C(N)=N \times I \cup a *(N \times 1), \quad C(M)=M \times I \cup b *(M \times 1)
$$

and extend $F$ conically to an embedding $F: C(N) \rightarrow C(M)$. By Theorem 2, there exists a PL-homeomorphism $h: C(M) \rightarrow C(M)$ such that $h \mid M \times 0$ is the identity and $h \hat{F}=\hat{F}$, where $\hat{F}$ is the standard conical extension of $F \mid N \times 0$. Note, in particular, $\hat{F}(C(N)) \cap(b *(M \times 1))$ $=\hat{F}(a *(N \times 1)) . b *(M \times 1)$ and $h(b *(M \times 1))$ are both relative regular neighborhoods of $\hat{F}(C(N)) \bmod \mathrm{Cl}(\hat{F}(C(N)-a *(N \times 1)))$ in 
the sense of Cohen [2]. By the uniqueness theorem [2], there is a PL-homeomorphism $k: C(M) \rightarrow C(M)$ such that $k \mid(M \times 0)$ $\cup \hat{F}(C(N))$ is the identity and $k(h(b *(M \times 1)))=b *(M \times 1)$. Let $H=(k h)^{-1} \mid M \times I$.

Example. If, in Theorem 3, the homomorphism $F_{*}: \Pi_{1}\left(N^{n} \times I\right)$ $\rightarrow \Pi_{1}\left(M^{n+1} \times I\right)$ is not an isomorphism, the conclusion is not necessarily true. Consider the following example. Let $\left(B^{n+2}, B^{n}\right)$ be a locally unknotted ball pair such that $\left(\partial B^{n+2}, \partial B^{n}\right)$ is knotted with $\Pi_{1}\left(\partial B^{n+2}-\partial B^{n}\right)$ not isomorphic to the integers. Let $K$ be a triangulation of $B^{n+2}$ containing $K \mid B^{n}$ and let $L$ be the 2nd derived neighborhood of $B^{n}$ in $K$. Let $v$ be a vertex in the interior of $B^{n}$. Let $N_{1}$ $=\partial\left(L \cap \partial B^{n+2}\right)$, which is PL-homeomorphic to $S^{n-1} \times S^{1}$. Let $N_{2}=\partial\left(\operatorname{Link}\left(v, K^{\prime \prime}\right) \cap \mathrm{Cl}\left(L-s t\left(v, K^{\prime \prime}\right)\right)\right)$ which is PL-homeomorphic to $S^{n-1} \times S^{1}$. Then $N_{1} \cup \partial\left(L \cap\left[B^{n+1}-\left(\partial B^{n+1} \cup \operatorname{star}\left(v, K^{\prime \prime}\right)\right)\right]\right) \cup N_{2}$ is a concordance between $N_{1}$ and $N_{2}$ but there cannot be an ambient concordance since $\Pi_{1}\left(\operatorname{link}\left(v, K^{\prime \prime}\right)-\operatorname{link}\left(v, K^{\prime \prime} \mid B^{n}\right)\right)$ is isomorphic to the integers.

REMARKs. Note that if one could prove Theorem 3 in the case when $n=3$, then all codimension one ball pairs unknot. All the theorems can be relativized to manifolds with boundary by considering proper embeddings with $n \geqq 6$.

\section{REFERENCES}

1. E. Akin, Manifold phenomena in the theory of polyhedra, $\mathrm{Ph} . \mathrm{D}$. thesis, Princeton University, Princeton, N. J., 1968.

2. M. M. Cohen, A general theory of relative regular neighborhoods, Trans. Amer. Math. Soc. 136 (1969), 189-229.

3. M. M. Cohen and D. Sullivan, On the regular neighborhood of a two-sided submanifold (to appear).

4. J. F. P. Hudson, Concordance and isotopy of PL embeddings. Bull. Amer. Math. Soc. 72(1966), 534-535.

5. - Piecewise linear topology. Vol. I, Mimeographed notes by J. L. Shaneson, University of Chicago, 1966/67.

6. L. S. Husch, On collapsible ball pairs, Illinois J. Math. 12(1968), 414-420.

7. W. B. R. Lickorish, The piecewise linear unknotting of cones, Topology 4(196566), 67-91.

8. W. B. R. Lickorish and L. C. Siebenmann, Regular neighborhoods and the stable range, Trans. Amer. Math. Soc. 139 (1969), 207-230.

9. J. W. Milnor, Whitehead torsion, Bull. Amer. Math. Soc. 72 (1966), 358-426.

10. J. B. Wagoner, Appendix I (P:S-Cobordism), Thesis, Princeton University, Princeton, N. J., 1966. 526.

11. E. C. Zeeman, Unknotting combinatorial balls, Ann. of Math. 78(1963), 501-

12. - Seminar on combinatorial topology, Mimeographed notes, Inst. Hautes Etudes Sci. Publ. Math., Paris, 1963. 